\title{
Novel Methods of Fabrication and Metrology of Superconducting NanoStructures
}

\author{
Ling Hao, John C. Macfarlane, John C. Gallop, David Cox, Patrick Joseph-Franks, \\ David Hutson, Jie Chen, Member, IEEE, and S. K. H. Lam
}

\begin{abstract}
As metrology extends toward the nanoscale, a number of potential applications and new challenges arise. By combining photolithography with focused ion beam and/or electron beam methods, superconducting quantum interference devices (SQUIDs) with loop dimensions down to $200 \mathrm{~nm}$ and superconducting bridge dimensions of the order $80 \mathrm{~nm}$ have been produced. These SQUIDs have a range of potential applications. As an illustration, we describe a method for characterizing the effective area and the magnetic penetration depth of a structured superconducting thin film in the extreme limit, where the superconducting penetration depth $\lambda$ is much greater than the film thickness and is comparable with the lateral dimensions of the device.
\end{abstract}

Index Terms-Magnetic field effects, nanotechnology, superconducting devices, thin films.

\section{INTRODUCTION}

$\mathbf{T}$ HE DEMANDS of metrology move toward ever more sensitive measurements on ever-smaller systems driven by industrial and scientific requirements. The dimensional metrology of thin-film structures with lateral dimensions in the range of $100 \mathrm{~nm}$ to $100 \mu \mathrm{m}$ and thicknesses in the range of $1-100 \mathrm{~nm}$ is a topic of increasing interest. Traditionally, superconducting devices such as superconducting quantum interference devices (SQUIDs) have been used at the macroscopic scale, but increasingly, there are potential applications at the nanoscale. These nanodevices can be used for a range of applications, including single-particle detection, spintronics, or quantum information processing. With the addition of appropriately configured antennas, applications in photon detection and spectroscopy at millimeter wavelengths or frequencies in the $\mathrm{THz}$ range become possible. In this paper, we describe how focusedion-beam (FIB) milling and/or electron-beam lithography can

Manuscript received July 11, 2006; revised October 5, 2006. This work was supported by the U.K. Department of Trade and Industry under the NMS QMP Projects QM04.3.2.

L. Hao, J. C. Gallop, and P. Joseph-Franks are with the National Physical Laboratory, TW11 0LW Teddington, U.K.

J. C. Macfarlane is with the Department of Physics, University of Strathclyde, G4 0NG Glasgow, U.K.

D. Cox is with the Nano Electronics Centre, Advanced Technology Institute, University of Surrey, GU2 7XH Guildford, U.K.

D. Hutson was with the Department of Physics, University of Strathclyde, G4 0NG Glasgow, U.K. He is now a self-employed Technology and Commercialization Advisor.

J. Chen is with the School of Engineering and Design, Brunel University, UB8 3PH Uxbridge, U.K.

S. K. H. Lam is with the Commonwealth Scientific and Industrial Research Organization Industrial Physics, Lindfield NSW 2070, Australia.

Color versions of one or more of the figures in this paper are available online at http://ieeexplore.iee.org.

Digital Object Identifier 10.1109/TIM.2006.890593 be combined with conventional photolithography to produce nonhysteretic SQUID devices having loop dimensions of the order $200 \mathrm{~nm}$ and superconducting bridge dimensions as small as $80 \mathrm{~nm}$. We describe the fabrication and characterization of these devices and demonstrate how they may be incorporated into measurement devices. We point out that for applications in nanoscale, single-layer thin-film SQUIDs are better suited than conventional devices incorporating trilayer Josephson junctions. Due to their intrinsically higher critical current densities and lower specific capacitances, microbridges permit the minimizing of both junction inductance and capacitance, which are essential to optimize SQUID energy sensitivity at the nanoscale [1]. To ensure that microbridges are close to ideal Josephson devices, it is necessary to deal with issues of adequate thermal shunting (through bridge geometry) and avoidance of hysteresis (through shunt-resistor fabrication) [2]. These issues will be outlined in Section II.

SQUIDs offer unique measurement capabilities for a wide range of physical parameters, from displacement to current or voltage. In this paper, we demonstrate the utility of nanoscale SQUIDs with reference to a specific example of the measurement of the effective superconducting penetration depth in a thin-film structure. Apart from practical difficulties encountered in properly defining their physical boundaries, the penetration of electromagnetic fields into superconducting structures presents a set of unavoidable additional problems for metrology applications. For example, complications arise since the penetration length $\lambda$ will vary strongly with temperature, frequency, and film thickness. We have developed a noninvasive in situ method to measure the temperature-dependent magnetic properties of ultrathin superconducting structures. We have employed this technique for characterizing effective inductance and penetration depth in the extreme limit, where $\lambda$ is much greater than the film thickness or even its lateral dimension. This method is applied to our devices, and we show that numerical and quasi-analytical methods show results that are in good agreement with the experiment.

\section{Film Deposition, Device Fabrication, AND TESTING}

We first emphasize that the magnetic properties of thin films and devices made from them are extremely sensitive to the deposition conditions and thickness of the films. Accurate dimensional control is possible only when the films themselves have been characterized, and as the relevant dimensions approach the submicrometer or nanometer scale, an in situ method 


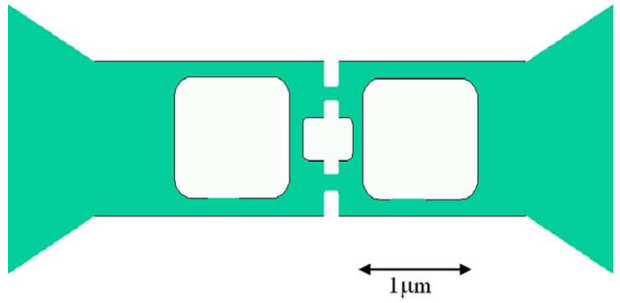

(a)

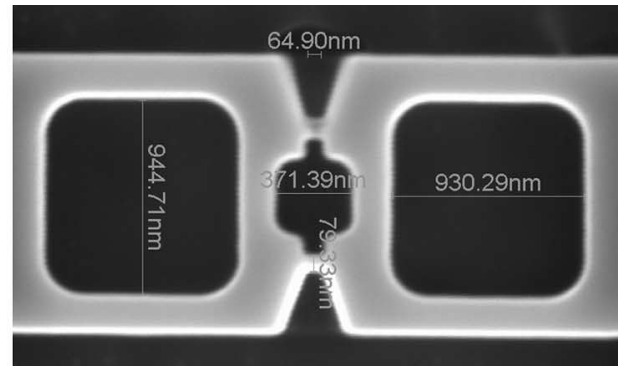

(b)

Fig. 1. (a) Schematic design of prototype superconducting structure with submicrometer dimensions. (b) SEM of actual device prepared from a Nb thin film by FIB milling.

is required to establish, for example, the magnetic penetration depth of the films used in a particular type of device. In this section, we therefore focus on the details of the film materials and the fabrication technique we have developed for preparing devices, which incorporate nanometer-scale features.

Crucially, our current application of FIB techniques has allowed the traditional photolithographic fabrication route to be retained and extended into the submicrometer and nanometer scale. Niobium is chosen as the basis of our low-temperature superconducting devices because of its long-established use, conveniently adjustable transition temperature $T_{c}(4.2-9.4 \mathrm{~K})$ [3], and the availability of suitable fabrication and clean-room facilities. Niobium films are deposited by dc magnetron sputtering from a $99.999 \%$ pure target onto oxidized silicon wafers or other suitable substrates (e.g., $\mathrm{MgO}$ and $\mathrm{Al}_{2} \mathrm{O}_{3}$ ) held near room temperature in an ultrahigh-vacuum chamber with a base pressure around $10^{-7} \mathrm{~Pa}$. By controllably varying the film thickness from 14 to $200 \mathrm{~nm}$, a range of $T_{c}$ from 5 to $9 \mathrm{~K}$ is achieved for the purposes of our experiment [4], [5].

For the SQUID devices used in this paper, Josephson junctions of microbridge type are used. In addition to the advantages previously noted, this design simplifies the fabrication process, compared with the traditional trilayer technique. The critical submicrometer bridge regions are individually prepared by FIB milling of the appropriate regions after the overall device structures such as the one shown in Fig. 1(a) have been delineated by photolithography and reactive-ion etching. A shunt resistor is formed by a 150-nm layer of tungsten hexacarbonyl, $\mathrm{W}(\mathrm{CO})_{6}$, which is deposited over the $\mathrm{Nb}$ tracks by using an electron beam. This layer prevents $\mathrm{Ga}$ ion implantation in the $\mathrm{Nb}$ junction during milling and subsequently acts as a suitable resistive shunt. During milling, simultaneous imaging of the sample with the electron beam is possible, allowing the milling to be stopped as soon as all the desired $\mathrm{Nb}$ is removed. To illustrate the capabilities of the technique, Fig. 1(a) shows a schematic design of a prototype structure incorporating submicrometer dimensions, whereas Fig. 1(b) shows a scanning electron micrograph (SEM) image of the completed device produced by FIB milling of a $\mathrm{Nb}$ thin film. A close-up of a bridge region is shown in Fig. 2. The superconducting characteristics of typical nanobridges made in this way are shown in Fig. 3. The critical current is about $240 \mu \mathrm{A}$ at a temperature of $8.75 \mathrm{~K}$. Note that there is no sign of hysteresis, and the microbridge junctions show near-ideal resistively shunted junction behavior.

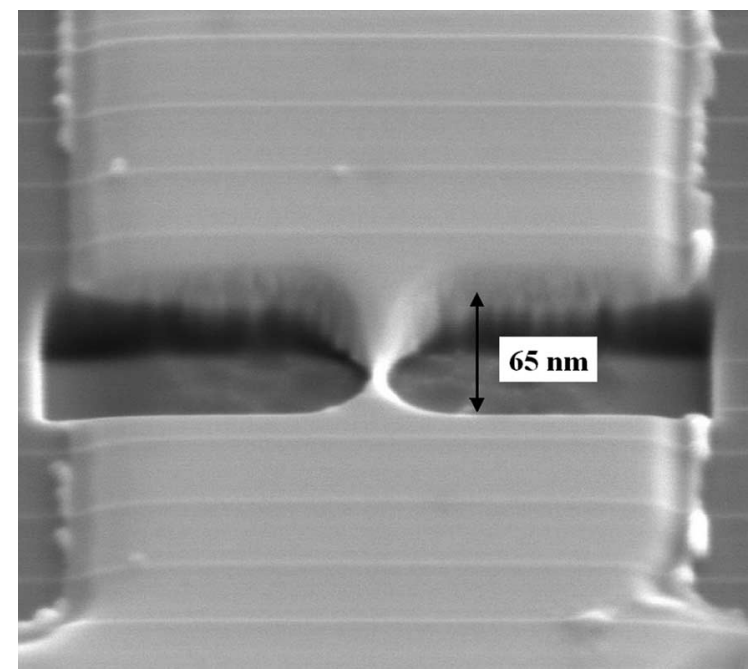

Fig. 2. Close-up SEM image of superconducting $\mathrm{Nb}$ nanobridge structure $\sim 80 \mathrm{~nm}$ wide produced by FIB milling.

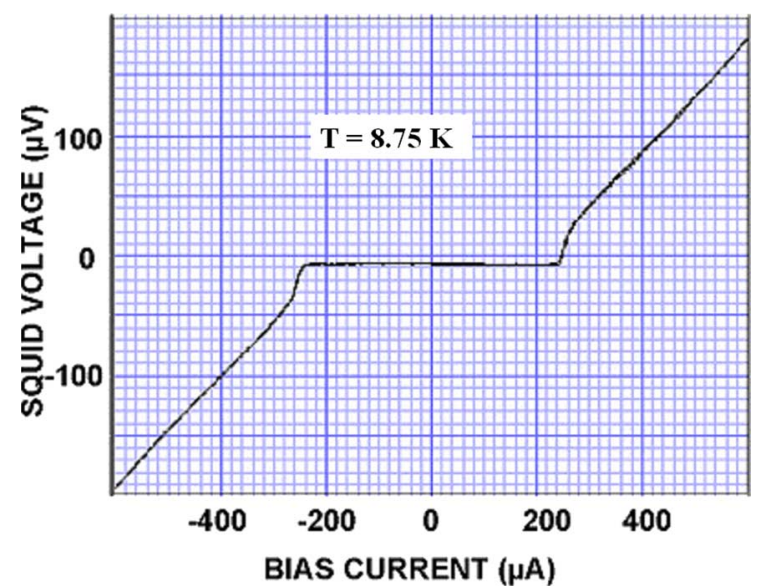

Fig. 3. $I-V$ characteristic for a SQUID containing two nanobridge junctions of the type shown in Fig. 2.

\section{Application of NANOSQUIDs to NoninVasive Measurement of Penetration Depth}

Measurements of the penetration lengths in thin superconducting films have usually been based on the two-coil method or variations thereof [6]-[8], where the lateral dimensions of 
the film are necessarily on the order of $10 \mathrm{~mm}$ due to the dimensions of practical wire-wound coils. Other techniques [9] are restricted to the high-frequency $(\mathrm{GHz})$ range. With a reduction of the dimensions of superconducting devices into the submicrometer regime (e.g., for quantum computing or single-particle counting applications), we have developed [10] a noninvasive in situ technique for measurement of the temperature-dependent magnetic properties of ultrathin superconducting structures. The method allows measurement of the effective area and penetration length in ultrathin-film structures of micrometer dimensions and can be extended to more general measurements of magnetic properties of nanoscale structures. It is based on the conventional definition of a SQUID's effective area $A_{\text {eff }}$, which is described as follows:

$$
A_{\text {eff }}=\Phi_{0} / \Delta B
$$

where $\Phi_{0}$ is the magnetic flux quantum, and $\Delta B$ is the change in applied field required to produce a response of one period in the SQUID's voltage versus field output. The technique is inherently broadband (dc to $\mathrm{MHz}$ ) in frequency.

We consider one of the simplest situations of interest: a plane lamina of radius $a$ and thickness $d$, which is exposed to a perpendicular dc magnetic field $B$. The structure has its superconducting normal transition at $T=T_{c 1}$. The London penetration depth $\lambda$ is such that $d<\lambda<a$. The 2-D (Pearl) length $\Lambda=\lambda^{2} / d$ is then comparable to or greater than the lateral dimension of the patch $a$ in the operating temperature range $0.5 T_{c 1}<T<T_{c 1}$. To simulate the application of a nanoscale SQUID making measurements on the thin-film structure, we note that the lamina is contained within a superconducting SQUID ring, which has a transition temperature $T_{c 2} \gg T_{c 1}$. Over the temperature range of interest, the ring remains fully superconducting. The calculations/modeling can be modified or extended to determine how the magnetic properties of the disk are affected by the presence of the ring.

For $T<T_{c 1}$, the effective area $A_{\text {eff }}$ and penetration length $\Lambda$ are related by the following equation (for a circular disk in this example, but other geometries can be similarly calculated):

$$
A_{\text {eff }}=\pi(a+2 \Lambda(T))^{2} .
$$

Rewriting this, we derive the effective penetration length $\Lambda$ and its temperature dependence from measurements of $A_{\text {eff }}$ as follows:

$$
\Lambda(T)=0.5\left((1 / \pi) A_{\mathrm{eff}}^{0.5}-a\right) .
$$

The SQUID-disk combination resembles a conventional SQUID at temperatures $T_{c 1}<T<T_{c 2}$, whereas at temperatures in the interval $0.5 T_{c 1}<T<T_{c 1}$, the effective area will increase with temperature up to some limit at which the disk (but not the surrounding SQUID ring) is entirely in a nonsuperconducting state.

In our experiments, the devices have been cooled in a wellshielded enclosure to liquid helium temperatures on a stage that can be temperature controlled in the range of $1.4-15 \mathrm{~K}$ or

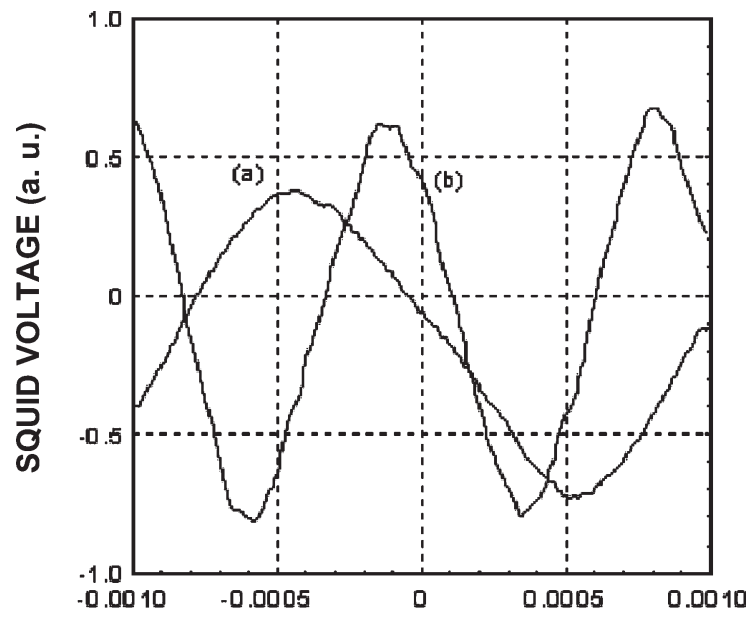

FIELD COIL CURRENT (A)

Fig. 4. Voltage response of a SQUID with an enclosed superconducting disk (see text) to an applied magnetic field (proportional to solenoid field current) at two different temperatures (a) $T=4.5 \mathrm{~K}$ and (b) $T=6.2 \mathrm{~K}$.

alternatively to as low as $100 \mathrm{mK}$ by using an adiabatic demagnetization refrigerator. The temperature stability is better than $0.005 \mathrm{~K}$. A 50 -turn field coil produces a magnetic field up to $50 \mu \mathrm{T}$ at the SQUID. Four terminal dc connections enable conventional $I-V$ and $V-\Phi$ characteristics to be measured by using a room-temperature preamplifier. For more sensitive measurement, an ac bias current at $49 \mathrm{kHz}$ is applied to the SQUID, and a low-temperature tuned transformer provides optimum coupling to the amplifier. In a typical experiment, a steadily increasing magnetic field is applied perpendicular to the plane of the SQUID, such that the SQUID's sinusoidal voltage-field characteristic undergoes an exact number of periods, as shown in Fig. 4. From repeated measurements, the mean value of $\Delta B$ corresponding to one flux period enables a mean value of $A_{\text {eff }}(T)$ to be evaluated from (1). By repeating the procedure at a range of temperatures, the corresponding temperature dependence of the penetration length $\Lambda(T)$ for the ultrathin superconducting patch is evaluated from (3). The flux response for two different temperatures is shown in Fig. 4. For comparison, identical SQUIDs that were fabricated without the internal superconducting patch showed no temperature-dependent change in effective area over the same temperature range. Note the large change in period between the two curves, demonstrating the strong contribution of the temperature-dependent Pearl effective penetration depth to the effective area of the SQUID loop. The effective area can increase by a factor of two as the temperature increases from $0.7 T_{c 1}$ to $0.95 T_{c 1}$. A typical set of our data, obtained by measurements of the SQUID's effective area, is compared with results of microwave transmission experiments [9] in Fig. 5. We note that the measured values of penetration length, ranging from $100 \mathrm{~nm}$ to $10 \mu \mathrm{m}$, can easily exceed the geometrical dimensions of typical structures such as those of Figs. 1 and 2. The present method allows us to make these measurements on thin-film samples just a few micrometers across, whereas the techniques employed by Wang et al. [3] and Gubin et al. [9], in common with others, required samples at least $10 \mathrm{~mm}$ in diameter. 


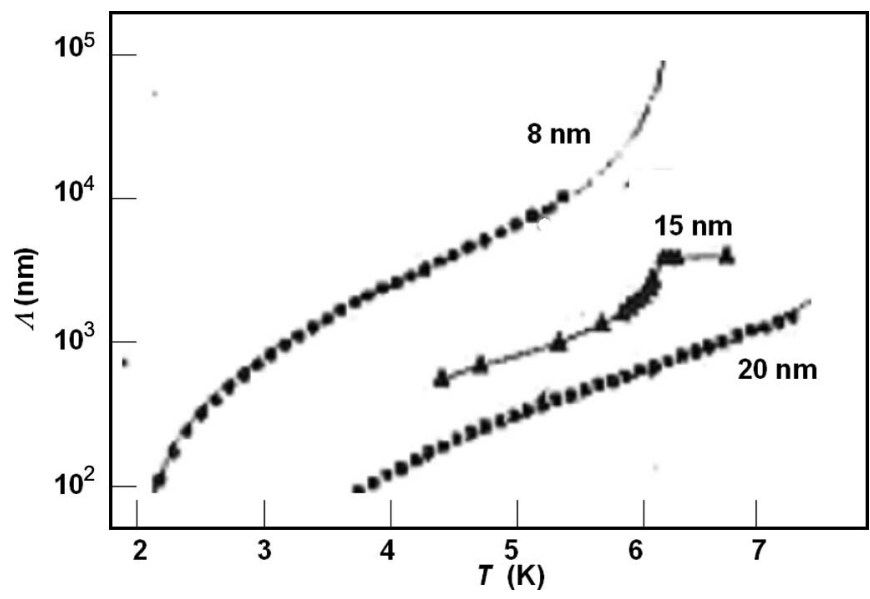

Fig. 5. Comparison of measured values of the temperature dependence of penetration length in $\mathrm{Nb}$ films ( $15 \mathrm{~nm}$ thickness, present technique) with results of a microwave technique (8 $\mathrm{nm}$ and $20 \mathrm{~nm}$ thickness, Gubin et al., [9]).

\section{Discussion AND CONCLUSION}

We aimed to show in this paper that SQUID technology has much to offer to the new science of nanoscale metrology. In order to address metrological needs at this important new length scale, it is essential first to solve a number of new fabrication problems. We have shown that the combination of electronbeam lithography or FIB milling with conventional photolithography can produce nanoscale microbridge weak links that have near-ideal Josephson properties. This procedure represents a much simpler and more appropriate solution to the problem of reduced physical scale than conventional trilayer processes can offer.

To illustrate the application of these nanoscale microbridge SQUIDs, we have demonstrated that the temperature dependence of the penetration depth of a thin superconducting film may be measured noninvasively, and that the technique covers a greater range both of physical dimensions and frequency bandwidth, compared with previous methods. The devices are robust, readily prepared, and characterized so that a wide range of metrological applications may be expected to follow.

\section{REFERENCES}

[1] J. Gallop, "SQUIDs: Some limits to measurement," Supercond. Sci. Technol., vol. 16, no. 2, pp. 1575-1581, Dec. 2003.

[2] S. K. H. Lam and D. L. Tilbrook, "Development of a niobium nanosuperconducting quantum interference device for the detection of small spin populations," Appl. Phys. Lett., vol. 82, no. 7, pp. 1078-1080, Feb. 2003.

[3] R. F. Wang, S. P. Zhao, G. H. Chen, and Q. S. Yang, "Absolute measurement of penetration depth in a superconducting film by the two-coil technique," Appl. Phys. Lett., vol. 75, no. 24, pp. 3865-3867, Dec. 1999.

[4] L. Hao, J. C. Gallop, C. H. Gardiner, P. Josephs-Franks, J. C. Macfarlane, S. K. H. Lam, and C. Foley, "Inductive superconducting transition-edge detector for single-photon and macro-molecule detection," Supercond. Sci. Technol., vol. 16, no. 2, pp. 1479-1482, 2003.

[5] L. Hao, J. C. Macfarlane, S. K. H. Lam, C. P. Foley, P. Josephs-Franks, and J. C. Gallop, "Inductive sensor based on nano-scale SQUIDs," IEEE Trans. Appl. Supercond., vol. 15, no. 2, pp. 514-517, Jun. 2005.

[6] E. H. Brandt and J. R. Clem, "Superconducting thin rings with finite penetration depth," Phys. Rev. B, Condens. Matter, vol. 69, no. 18, pp. 184509.1-184509.12, May 2004.

[7] A. T. Fiory, "Penetration depths of high Tc films measured by two-coil mutual inductances," Appl. Phys. Lett., vol. 52, no. 25, pp. 2165-2167, 1998.
[8] J. H. Claassen, J. E. Evetts, R. E. Somekh, and Z. H. Barber, "Observation of the superconducting proximity effect from kinetic-inductance measurements," Phys. Rev. B, Condens. Matter, vol. 44, no. 17, pp. 9605-9608, Nov. 1991.

[9] A. I. Gubin, K. S. Il'in, S. A. Vitusevich, M. Siegel, and N. Klein, "Dependence of magnetic penetration depth on the thickness of superconducting $\mathrm{Nb}$ thin films," Phys. Rev. B, Condens. Matter, vol. 72, no. 6, p. 064503 , Aug. 2005.

[10] L. Hao, J. C. Macfarlane, J. C. Gallop, and S. K. H. Lam, "Direct measurement of penetration length in thin and/or mesoscopic superconducting structures," J. Appl. Phys., vol. 99, no. 12, p. 123916, Jun. 2006.

Ling Hao received the B.Sc. degree in general physics and the M.Sc. degree in solid state physics from Beijing Normal University, Beijing, China. She received the Ph.D. degree from the Department of Physics and Applied Physics, University of Strathclyde, Glasgow, U.K., for research on electronic noise in superconducting devices in 1995.

Since then, she has been with the National Physical Laboratory, Teddington, U.K. Her current research interests include the development of develop novel applications of superconductors (e.g., nano-SQUIDs) and nanoscience (e.g., carbon nanotubes) to precision measurement. She is a lead scientist in areas including single-particle detection, microwave and optical spectroscopy, and quantum thermal metrology at the nanoscale.

Dr. Hao is a member of the Institute of Physics, U.K., and a Chartered Physicist.

John C. Macfarlane received the B.Sc. degree from University of Glasgow, Glasgow, U.K., and the Ph.D. degree from the University of Strathclyde, Glasgow, in 1958 and 1964, respectively.

During the next 27 years, he was with the Commonwealth Scientific and Industrial Research Organization, Lindfield, Australia, working initially on the atomic hydrogen maser frequency standard and later joining the Josephson Voltage Standard team in 1969. He subsequently worked in the general area of superconducting electronics, becoming Leader of the High Tc Superconductivity Group in 1986. He returned to Scotland in 1992 as a Visiting Professor at the University of Strathclyde and is currently a Senior Research Fellow. Since 1996, his work has included collaborative research with the Quantum detection group at the National Physical Laboratory, Teddington, U.K.

Dr. Macfarlane is a Fellow of the Australian Institute of Physics and the Institute of Physics, U.K.

John C. Gallop was born in Bristol, U.K., in 1943 and received the B.A. and Dr. Phil. degrees from Oxford University, Oxford, U.K., in 1965 and 1970, respectively.

Since then, he has been with the National Physical Laboratory (NPL), Teddington, U.K., working on the metrological applications of superconductivity and, more recently, of nanoscience. He is currently involved in exploiting scanned probe microscopy techniques, microwave properties of cryogenic resonators, as well as developing HTS SQUIDs for precision measurement. He recently retired but continues to work part-time at NPL and remains to be a Visiting Professor at Imperial College, London, U.K.

Dr. Gallop is a Fellow of the Institute of Physics, U.K.

David Cox received the Ph.D. degree from the Department of Materials Science and Metallurgy, University of Cambridge, Cambridge, U.K., in 2001.

Subsequently, he has worked in the Advanced Technology Institute, University of Surrey, Guildford, U.K., and in 2005, was appointed as a Visiting Strategic Research Fellow at the National Physical Laboratory, Teddington, U.K. He currently specializes in nanofabrication using FIB and electron microscope in situ manipulation methods. 
Patrick Joseph-Franks was born in Bristol, U.K., in 1960. He received the B.Sc. degree in physics with astronomy and was awarded the Dr. Phil. degree for work on A Helium-3 Magnetometer for the Neutron Electric Dipole Moment Experiment from the University of Sussex, Sussex, U.K., in 1986.

He then joined the U.K. Ministry of Defence, developing a gyroscope based on nuclear spin of helium-3 nucleii and also on high temperature superconductors and SQUID detectors. In 1991, he joined the National Physical Laboratory (NPL), Teddington, U.K. He is a Principal Research Scientist in the Fundamental and Wavelength Standards Team, NPL. He has managed over four Department of Trade and Industry funded projects at NPL worth in excess of $\$ 1.5 \mathrm{M}$. His main interests include nanoengineered structures using ultrahighvacuum scanning tunneling microscopy, the development of miniature SQUIDs for single-spin detection, and electron and nuclear spin resonance.

$\mathrm{He}$ is a member of the Institute of Physics, U.K., and a Fellow of the Royal Astronomical Society.

David Hutson was born in Doncaster, U.K., in 1960. He received the B.Sc. degree in applied physics and the Ph.D. degree in physics and applied physics from the University of Strathclyde, Glasgow, U.K., in 1981 and 1987, respectively.

From 1985 to 1991 and from 1996 to 2000, he worked with leading manufacturers of data storage devices with responsibilities for the research and development of recording-head components utilizing thin-film technologies. In the interim period, he returned to the academia and was responsible for the successful development of a superconducting gradiometer for the detection of neurological activity in the brain and spinal cord. During 2001-2003, he managed a start-up company specializing in the development of novel thinfilm materials for sensors and semiconductor applications. He is currently an independent Technology and Commercialization Advisor, assisting universities and businesses with sensor design, process development, technology transfer, and commercialization projects.
Jie Chen (S'93-M'95) received the Ph.D. degree from the University of York, York, U.K., in 1995.

He joined Brunel University, Uxbridge, U.K., as a Lecturer, in February 1998. Before that, he was with the University of Hull, Hull, U.K.; the University of Strathclyde, Glasgow, U.K.; and the University of York, as a Lecturer/Research Associate. He has worked in the field of modeling and control of dynamic systems for many years and has published more than 60 papers in international journals and conference proceedings. $\mathrm{He}$ is an Associate Editor of the International Journal of Systems Science. His current research interests include the dynamics, control, and application of mathematical modeling and numerical methods to a wide range of problems.

Dr. Chen was awarded the 1997 IEE Kelvin Premium for a paper published in the IEE Proceedings Part D.

S. K. H. Lam received the Ph.D. degree from the University of Sydney, Sydney, Australia, in 2000

He is currently with the Commonwealth Scientific and Industrial Research Organization, Lindfield, Australia, as a Research Scientist. His main research interests include superconducting material and junction technology, SQUID, nanostructure fabrication, and magnetism on nanoparticle and molecules. 